\title{
A REVIEW ON NEXT GENERATION MOBILE COMMUNICATION-5G
}

\author{
NANDHAKUMAR P. \\ Engineer, P3 Communications, Bangalore, India \\ E-mail: pnandha92@gmail.com
}

\begin{abstract}
In the upcoming years (expected 2020), 5G technology is going to deploy all around the world. Presently a lot of researches and project on the fifth generation mobile communication system are finalized and tested successfully in the research labs. Within two to three years, $5 G$ technology is going to replace the present technology and many mobile gadgets are coming with $\mathbf{5 G}$ support. The main focus of this technology is to provide better spectrum utilization, ultra speed communication, QoS, etc. In this article, the comprehensive analysis of next-generation mobile communication system is provided and it includes technologies employed for 5G. This work will give a detailed investigation and discussion on the features of $5 G$ technology.
\end{abstract}

Keywords: 5G Spectrum Allocation, 5G Key Technologies, 5G Projects.

\section{INTRODUCTION}

Day by day the consumption of data and user devices are increasing exponentially, this may result in bandwidth consumption in the coming years [1, 2]. Presently, telecommunication industries are confronting a set of problems, to provide high speed and uninterrupted services to the user because of the usage of internet and voice over data are increasing now a daytime. The demands from the users of the network services are increasing day by day. To meet the user demands, the idea of 5G technology evolved [2]. A lot of researches are going on the implementation of the 5G technology and many projects are already completed successfully, which may deploy all around the world within two to three years. The aim of this technology is to provide a better Quality of Service (QoS) as compared with the present $3 \mathrm{G}$ and $4 \mathrm{G}$ technology [3]. The five important features expected from the $5 \mathrm{G}$ technology are better spectrum utilization, $24 \times 7$ uninterrupted services, ultra speed gigabit connection, zero latency, and coverage. The present $3 \mathrm{G}$ and $4 \mathrm{G}$ technologies are playing an important role in the mobile communication system. Code Division Multiple Access (CDMA) and Orthogonal Frequency Division Multiplexing (OFDM) are the popular and present ruling techniques in $3 \mathrm{G}$ and $4 \mathrm{G}$ technology respectively. CDMA, WCDMA, and UMTS are the multiple access $3 \mathrm{G}$ technology that provides high data rate communication to the users [4]. Using this technology, the information from the base station transmits to the multiple users over the same channel with the help of spreading codes. Hence this technology is popularly known as spread spectrum technology. Even today this technology is running successfully in many countries because of its low transmitting power and wider bandwidth. The primary disadvantage of this technology is Inter-Symbol Interference (ISI) and bandwidth wastages that lead to the OFDM modulation technique which is known as 4G technology. In 2010, this technology adopted globally and it is successfully running in many countries. OFDM is the multi-carrier modulation technique, the multiple numbers of information will be modulated with the multiple subcarriers and it will be transmitted over the channel. So as compared with the previous technology, 4G OFDM technique achieved a greater throughput. One of the significant advantages of this technique is "No ISO" because of the sub-carriers, which is orthogonal to each other and the wider bandwidth is split into narrow bands [5]. This technique is presently used in the 4G LTE system and WiMax system, but the Cyclic Prefix (CP) present in the OFDM symbols, creating a spectral loss because of repeating the same symbols and it affects the throughput of the system. $\mathrm{CP}$ is playing a really significant role in OFDM technique to reduce the ISI. Without CP insertion, the OFDM technique is not possible. In order to reduce the spectral loss, need to look for a new modulation technique that leads to nextgeneration technology. There are lots of new modulation techniques are already planned for the next generation mobile communication system, Filter Bank Multicarrier (FBMC) is the new and advanced modulation technique where CP is not applied [6]. This is one of the techniques expected to integrate into the $5 \mathrm{G}$ system to achieve the best spectrum utilization. The detailed discussion of new modulation technique, key technologies, projects and challenges for $5 \mathrm{G}$ technologies are explained in the following section.

\section{5G SPECTRUM ALLOCATION}

Allocating the spectrum for the next generation mobile communication system is the challenging task for the research community and the industries. The idea of allocating the new frequency band for $5 \mathrm{G}$ technology coming from the experience of spectrum scarcity in the present mobile frequency bands. From the study of IMT research, the range of $5 \mathrm{G}$ frequency bands will be considered from $1 \mathrm{GHz}$ to $100 \mathrm{GHz}$, the range will be extended up to $275 \mathrm{GHz}$ in future [1]. Globally $5 \mathrm{G}$ frequency bands are split into two parts, frequency below $6 \mathrm{GHz}$ and frequency above $6 \mathrm{GHz}$. The frequency below $6 \mathrm{GHz}$ is going to be the best resource in the near future [7]. $5 \mathrm{G}$ frequency band specification and definition is 
given by the 3GPP standard in Dec 2017. These frequency bands are $3.3-4.2 \mathrm{GHz}, 4.4-5 \mathrm{GHz}, 24.25$ $29.5 \mathrm{GHz}$ and $37-40 \mathrm{GHz}$. It also includes some lowfrequency old bands below $3 \mathrm{GHz}$. The WRC-19 (Al 1.13) candidate bands under study [7,8] are shown in Table 1.

Table.1 WRC-19 (Al 1.13) Frequency bands under study

\begin{tabular}{|l|l|l|}
\hline \multicolumn{3}{|c|}{ WRC-19 Frequency Bands (GHz) } \\
\hline $24.25-27.5$ & $47.2-50.2$ & $31.8-33.4$ \\
\hline $37-40.5$ & $50.4-52.6$ & $40.5-42.5$ \\
\hline $42.5-43.5$ & $66-76$ & $47-47.2$ \\
\hline $45.5-47$ & $81-86$ & \\
\hline
\end{tabular}

Some bands below $6 \mathrm{GHz}$ are going to use for the early phase of $5 \mathrm{G}$ deployment. Frequency below $6 \mathrm{GHz}$ proposed for $5 \mathrm{G}$ in different countries are Europe (3.4$3.8 \mathrm{GHz}$ ), Japan (3.6-4.2GHz), South Korea (3.4$3.7 \mathrm{GHz})$ and China $(3.3-3.6 \mathrm{GHz})$. The frequency below $1 \mathrm{GHz}$ bands is also proposed for $5 \mathrm{G}$ because of its wide area coverage capability and low implementation cost. The European Commission has chosen $700 \mathrm{MHz}$ band for the indoor communication purpose and the USA also focuses on $600 \mathrm{MHz}$ band [7]. So the bands below $1 \mathrm{GHz}$ are also proceeding to integrate into the $5 \mathrm{G}$ spectrum. The high-frequency bands above $6 \mathrm{GHz}$ are also proposed and tested in many countries [9]. Recently South Korea tested $26.5-29.5 \mathrm{GHz}$ band at the Winter Olympic Games, conducted in Feb 2018 [7].

\section{KEY TECHNOLOGIES IN 5 G}

The features and key technologies for 5G mobile communication technology are explained in the following section.

\subsection{Filter Bank Multicarrier (FBMC)}

FBMC is one of the multicarrier modulation techniques; it is the advanced version of the OFDM technique, proposed for the next generation mobile communication system. This technique has been evolved to overcome the drawbacks of OFDM system, especially for the spectral efficiency [6]. The significant advantages of this technique are No CP, lower spectral side lobs and less out-of-band leakage. As compared with the current OFDM system, FBMC offers many advantages and it is considered to be a perfect solution in minimizing the adjacent band interference $[5,10]$. So FBMC is the required candidate for the $5 \mathrm{G}$ mobile communication.

\subsection{Universal Filtered Multicarrier (UFMC)}

OFDM is the most prominent and successful running technique in the current 4G LTE system. Although this technique has some drawbacks. The first and main drawback of this technique is spectrum efficiency loss because of the CP insertion [1]. Secondly, spectral leakage. The current $4 \mathrm{G}$ technology is functioning on the below $6 \mathrm{GHz}$ frequency bands. when it goes for a higher frequency band $(5 \mathrm{G})$ above $6 \mathrm{GHz}$ [7], the spectral leakage and efficiency will affect to a greater extent. To overcome this problem, a new waveform has been studied and proposed for $5 \mathrm{G}$. One of the techniques is known as UFMC, it is also the multi-carrier technique, where the filter is applied to a group of sub-carriers unlike FBMC or OFDM [11]. The performance of the system will increase because it reduces the side lobes. Also, this technique is best suitable to integrate with MIMO technology.

\subsection{Generalized Frequency Division Multiplexing (GFDM)}

GFDM is the novel candidate for a next-generation communication system. It is a non-orthogonal method; carriers are not orthogonal to each other. However, the Inter Symbol Interference (ISI) and Inter-Carrier Interference (ICI) are removed with the help of near orthogonality concepts (GFDM/OQAM) [12]. The primary motive of this technique is to reduce Pear Average Power Ratio (PAPR) and Out of Band (OOB) emission. The advantages of GFDM are reduced PAPR, less complex in signal processing and reduced OOB [13]. To improve the transmission rate and Bit Error Rate (BER), it is necessary to integrate GFDM with MIMO technology.

\subsection{Beam Division Multiple Access (BDMA)}

BDMA is a new multiple access transmission technology, where Base Station (BS) communicates with Mobile Stations (MS) through the focused beam [1]. This technology is constructed on the multiple antenna concepts (MIMO/Massive MIMO), a massive number of antennas at BS will focus on each individual MS user for the communication. Each individual user will be selected according to the user scheduling concept. According to the position of the MS users, the direction and width of the beam will be calculated by the BS. Massive MIMO BDMA technology is going to be a novel candidate for 5G networks [14].

\subsection{Massive MIMO Communication.}

One of the main targets of 5G technology is to increase the throughput of more than a thousand times as compared with the present throughput of the system. Higher throughput can be achieved by increasing the spectral efficiency (bits/Hz/cell) [15]. This can be achieved by placing multiple numbers of antennas at both BS and MS; this technology is known as MIMO/Massive MIMO [1]. There are three main important factors considered to increase the throughput of the system are bandwidth, cell density, and spectral efficiency. Massive MIMO is a multi-user MIMO, where multiple numbers of antennas at $\mathrm{BS}$ and $\mathrm{N}$ number of users per BS. This technique was proposed by Dr. T.L Marzetta and received many scientific awards [16]. This technology was proposed not only for the throughput 
improvement, but it will also improve the spectral efficiency.

\subsection{Millimeter Wave Communication}

The demands for the higher data rate communication and QoS are increasing day by day. $5 \mathrm{G}$ is the expected technology and capable of connecting billions of devices (Smart Phones and Tablets) in the future [17]. Plenty of research activities in millimeter wave communication are going on because of its ultra-wide spectrum availability. This technology was proposed especially for indoor or small cell based communication. The propagation losses will increase with the increase of distance travel, because of its higher frequency [18]. This loss can be reduced by equipping multiple antennas at both millimeter wave BS (mBS) and MS [17]. Multiple bands from $1 \mathrm{GHz}$ to $10 \mathrm{GHz}$ are allocated for this communication and different waveforms such as Single Carrier OFDM (SCOFDM), Filtered OFDM (F-OFDM) and Sparse Code Multiple Access (SCMA) can be used for $5 \mathrm{G}$ millimeter wave communication [17].

\subsection{Full Duplex Communication for 5G}

Many researchers proposed a full-duplex operation for the 5G communication system. The aim of this technology is to provide improved spectral efficiency as compared with the half-duplex or Out-of-Band Full Duplex (OBFD) [19]. In-Band Full Duplex (IBFD) communication is the popular duplex operation, which enables improved spectral efficiency and transmission over the same frequency band [20]. This is the real challenging task to the researchers. This concept can be implemented in the Medium Access Control (MAC) layer, meaning that at the same time the terminal can transmit and received the frames continuously $[19,20]$.

\subsection{Device to Device Communication (D2D)}

D2D communication is the communication between the end user devices (MS) with the absence of BS. This technology proposed for the 5G short distance communication purpose [21]. Users can switch $\mathrm{ON}$ the direct communication link and bypass the BS, if the users are close to each other. Suppose the users are not in the D2D range, then users can switch to BS for the communication. There are three important advantages in this technology are energy efficient, spectral efficiency and improved data rate. D2D communication classified into two types, they are in-band and out-band communication. Already this concept implemented by Qualcomm in the name of flashlinq architecture [21].

\subsection{Spectrum Sharing for $5 G$}

Spectrum scarcity is one of the important issues in the present cellular environment, because of the rapid consumption of spectrum resources. To rectify this issue, need to find a new spectrum, particularly in the higher frequency bands [1]. The present $2 \mathrm{G} / 3 \mathrm{G} / 4 \mathrm{G}$ networks are designed for licensed spectrum, meaning that only licensed users can access this spectrum. In the future, unlicensed users can also access the licensed user's spectrum and vice versa with the help of spectrum sharing techniques (Dynamic Spectrum Access) [2, 5]. There are many spectrums sharing techniques proposed for $5 \mathrm{G}$, they are Coordination method, GLDB support, and Cognitive Radio Dynamic Spectrum Access (CRDSA) Method. CRDSA is a popular and well-known spectrum access technology, proposed by Dr. Joseph Mitua. The two primary steps in CR are spectrum sensing and spectrum sharing. Spectrum sensing is the initial step in this technology; it will sense the entire spectrum and detect the free or unused spectrum [5]. Secondly, it will share that unused spectrum to the user, who request to access the spectrum. This is a possible and efficient way to utilize the un-crowded spectrum.

\subsection{Visible Light Communication for 5G-LIFI}

Visible light communication is optical based mobile communication technology. This technology is especially proposed for ultra speed access [1]. The spectrum considered for this technology is from $400 \mathrm{THz}$ to 490THz (Visible light spectrum) bands. The primary targets of this technology are ultra speed and high secured communication access. This technology is best suitable for indoor attocell environment and it contains the Central Control Unit (CCU), Optical BS (OBS) and Optical fiber backhaul connection. The coverage area of each OBS is very small, attocell coverage [22]. It uses hybrid LED's and detectors for transmission and reception respectively.

\subsection{Femto Cell}

Femto cell is the novel and innovative cell design for indoor communication. From the study, a number of users are available and using the network in the indoor area only [1], meaning that the number of voice and data usages are mostly arisen from the indoor area as compared with the outdoor area, but the coverage is very less in indoor areas. Femto cell is the best suitable candidate for the 5G technology; it will solve the less coverage issue by equipping Femto $\mathrm{BS}$ in the indoor environment (Offices, Shopping Malls, and Universities) [1].

\section{5G PROJECTS}

5GPPP- 5GPPP (5G Infrastructure Public Private Partnership) is the European Commission (EU) project. In June 2017, new twenty-one projects were launched in Brussel. 5GPPP focuses on next-generation wireless communication infrastructure. The primary objective of this project is to provide a capacity improvement as compared with current 4G capacity, energy saving, secure communication, and 24x7 uninterrupted ultra speed communications to the users. The upcoming 
5GPPP focused on 5G-LTE, cm-Wave and mm-Wave communication [23].

5G Brazil- 5G Brazil is the telebrazil umbrella project. It is associated with many institutions, industries, research centers, and network operators. The main objective of this project is to develop the $5 \mathrm{G}$ network system throughout in Brazil [24].

5G MF- 5G MF (5G Mobile Communication Promotion Forum) is the new project associated with Radio Industries and Business (Japan), started in Sep 2014. The main aim of this project is to provide a high speed, high capacity, Massive device communication, ultra-low latency, ultra-high reliability, energy efficiency and cost saving [25].

5G Americas- 5G Americas is an industry organization associated with network providers and manufacturers. Some of the popular companies associated with this organization are Cisco, Intel, Nokia, Qualcomm, and Samsung. The mission of this organization is to develop 5G and LTE wireless technology [26].

IMT-2020 (5G) - IMT 2020 group started in Feb 2013 and it is established by the Ministries if China. This group associated with many research institutions and it is conducting much $5 \mathrm{G}$ research for the development for China network [27].

5G PICTURE- 5G PICTURE (5G Programmable Infrastructure Converging Network and Compute Research) project started in Jan 2017. The duration of this project is about two years. The aim of this project is to develop the front and backhaul infrastructure for both the wireless and optical networks [28].

5GIC- 5GIC (5G Innovative Centre) is the UK research Centre, running by University of Surrey. The vision of $5 \mathrm{GIC}$ is to develop the infrastructure for $5 \mathrm{G}$ wireless communication. This Center is associated with many industries and academics. The research work focuses on the Internet of Thing (IoT), cellular and satellite communication [29].

\section{CONCLUSION}

This article deals with the comprehensive study and discussion on the 5G technology. The projects, spectrum allocations and key technologies for $5 \mathrm{G}$ networks are investigated and discussed. From the discussion, 5G technology is the only potential path to overcome the present obstacles such as spectrum scarcity, high energy consumption, and implementation complexity. New modulation technique, new cell concepts, and multiple antenna systems are discussed in this article for the next generation of mobile communication. In 2020, 5G technology is expected to deploy all around the world and it will bring a revolutionary change in the field of wireless communication.

\section{ACKNOWLEDGMENTS}

Authors like to thank Mr. Mosin Naragund (Engineer in RCIL) for his support and valuable suggestions.

\section{REFERENCES}

[1] Kumar. A, Gupta. M, "A review on activities of fifth generation mobile communication system", Alexandria Engineering Journal, Elsevier, pp-1-11, Feb 2017.

[2] Jiang D and Liu G -An overview of $5 \mathrm{G}$ requirements5G mobile communications, Spinger, pp. 3-26, 2017. DOI: 10.1007/978-3-319-34208-5_1.

[3] Zhang. P, Lu. A, Wang. Y, Wang. Q, "Cooperative localization in 5G networks: A survey", ICT Express, Elsevier, 3(1), pp.27-32, Mar 2017.

[4] Kumar. A, Gupta. M, "Design, comparative study and analysis of CDMA for different modulation techniques", Egyptian Informatics Journal, Elsevier, Vol. 16, No.3, pp. 351-365, 2015.

[5] Pandi. N, Kumar. A, "A Review on Cognitive Radio for Next Generation Cellular Network and its Challenges", American Journal of Engineering and Applied Sciences, Vol.10, No.2, pp. 334-347,April 2017

[6] Jayaprakash. A \& Reddy. G, “ Discrete Ambiguity Function Based Analysis of Filter Bank Multicarrier Systems", IETE Technical Review, 2015. DOI: 10.1080/02564602.2015.1015941.

[7] Jee.J, et al, "Spectrum for 5G: Global Status, Challenges, and Enabling Technologies", IEEE Communications Magazine, IEEE, 56(3), pp.12-18, March 2018.

[8] https://www.itu.int/en/ITU-R/seminars/rrs/RRS-17 Americas/Documents/Forum/9_ITU\%20Diana\%20 Tomimura.pdf.

[9] Tan. Wang, Gen. Li,- Spectrum Analysis and Regulations for 5G-5G mobile communications; Spinger, pp. 27-50, 2017. DOI: 10.1007/978-3-31934208-5_2.

[10] Mattera. D, Tanda. M, Bellanger. M- New Multicarrier Modulations for 5G-5G mobile communications; Spinger, pp. 165-202, 2017. DOI: 10.1007/978-3-319-34208-5_7.

[11] K. Zerhouni, et al, On the Cyclostationarity of Universal Filtered Multi-Carrier UFMC, International Journal of Electronics and Communications, 2018, doi: https://doi.org/10.1016/j.aeue.2018.03.016.

[12] Y-K. Chang, F-B. Ueng, A Novel Turbo GFDM-IM Receiver for MIMO Communications, International Journal of Electronics and Communications, 2018, doi: https://doi.org/10.1016/j.aeue.2018.01.021.

[13] Matthe. M, et al,- Generalized Frequency Division Multiplexing: A Flexible Multi-Carrier Waveform for 5G-5G mobile communications; Spinger, pp. 223-257, 2017. DOI: 10.1007/978-3-319-342085_9. 
[14] Choi, Yong I, et al, "On the Performance of Beam Division Nonorthogonal Multiple Access for FDDbased Large-scale Multi-user MIMO Systems", IEEE Transactions on Wireless Communications,Vol.16, No.8, pp. 5077 - 5089, 2017.

[15] Trinh Van Chien, Emil Björnson- Massive MIMO Communications-5G mobile communications; Spinger, pp. 77-116, 2017. DOI: 10.1007/978-3319-34208-5_4.

[16] https://www.bell-labs.com/usr/tom.marzetta.

[17] Wang. Y, Shi. Z- Millimeter-Wave Mobile Communications-5G mobile communications; Spinger, pp. 117-133, 2017. DOI: 10.1007/978-3319-34208-5_5.

[18] Bogale, Endeshaw T, Le. L, "Massive MIMO and mmWave for $5 \mathrm{G}$ wireless HetNet: Potential benefits and challenges." IEEE Vehicular Technology Magazine, Vol.11, No.1, pp.64- 75, 2016. DOI: 10.1109/MVT.2015.2496240.

[19] Mingxin Zhou, Yun Liao, and Lingyang Song, "Full-DuplexWireless Communications for 5G", 5G mobile communications; Spinger, pp.229-336, 2017. DOI: 10.1007/978-3-319-34208-5_11.

[20] Sanharwal A, et al, "In-Band Full-Duplex Wireless: Challenges and Opportunities", IEEE Journal on Selected Areas in Communications, 32(9), pp. 0733-8716, 2014.

[21] Araniti. G, "Device-to-Device Communications over 5G Systems: Standardization, Challenges and Open Issues", 5G mobile communications; Spinger, pp. 337-360, 2017. DOI: 10.1007/978-3-319-342085_12.

[22] Harald Haas , LiFi is a Paradigm-Shifting 5G Technology, Reviews in Physics, 2017, doi: 10.1016/j.revip.2017.10.001.

[23] 5GPPP, https://5g-ppp.eu/projects/.

[24] 5G Brazil, https://5g-ppp.eu/global-5g-actions/.

[25] 5G-MF, http://5gmf.jp/en/.

[26] 5G Americas, www.5gamericas.org.

[27] IMT 2020 (5G), http://www.imt-2020.cn/en.

[28] http://www.bristol.ac.uk/engineering/research/ csn/ projects/current/picture/

[29] https://www.surrey.ac.uk/5gic. 